INPLASY

PROTOCOL

To cite: Santos et al. Chronic effect of hip thrust exercise compared with control or squat on performance of soccer players: A systematic review and meta-analysis of randomized controlled trials. Inplasy protocol 202140005. doi:

10.37766/inplasy2021.4.0005

Received: 01 April 2021

Published: 02 April 2021

Corresponding author: Jonatas Ferreira da Silva Santos

jonatas_contato@hotmail.com

Author Affiliation:

Federal University of the Jequitinhonha and Mucuri Valleys

Support: Ministério da Cidadania SNFDT.

Review Stage at time of this submission: Piloting of the study selection process.

Conflicts of interest:

None declared.

\section{Chronic effect of hip thrust exercise compared with control or squat on performance of soccer players: A systematic review and meta-analysis of randomized controlled trials}

Santos, JFS1; Martins, ML2; Amorin, FT3; Peixoto, MFD4; Gripp, F4.

Review question / Objective: What are the effects of hip thrust interventions on strength, power, agility and speed among soccer players when compared to a control group?

Condition being studied: Chronic interventions involving hip thrust or barbell hip thrust exercise in soccer players from any sex or age.

Information sources: Eletronic databases (PubMed, Web of Science, Scopus and Google scholar) were searched for relevant publications.

INPLASY registration number: This protocol was registered with the International Platform of Registered Systematic Review and Meta-Analysis Protocols (INPLASY) on 02 April 2021 and was last updated on 02 April 2021 (registration number INPLASY202140005).

\section{INTRODUCTION}

Review question / Objective: What are the effects of hip thrust interventions on strength, power, agility and speed among soccer players when compared to a control group?

Rationale: Hip thrust exercise can be used to improve soccer player's athletic 
performance, including strength, sprint, change of direction and jump. However, a systematic review regarding the effect of hip thrust exercise on soccer player's is lacking.

Condition being studied: Chronic interventions involving hip thrust or barbell hip thrust exercise in soccer players from any sex or age.

\section{METHODS}

Search strategy: Eletronic databases (PubMed, Web of Science, Scopus and Google scholar) were searched for relevant publications. Keywords and synonyms were entered in various combinations in all fields: (soccer OR soccer player OR football OR football player) AND (hip thrust OR barbell hip thrust).

Participant or population: Apparently healthy soccer players, with no restrictions on their playing level, sex, or age.

Intervention: Chronic hip thrust-based programmes.

Comparator: Passive or active control groups.

Study designs to be included: Controlledtrial.

Eligibility criteria: Inclusion criteria: (i) Apparently healthy soccer players, with no restrictions on their playing level, sex, or age; (ii) Chronic HT-based programmes; (iii) Active control group; (iv) Pre-post intervention values of máximal strength, power, sprint, change of direction and jump; (v) controlled and/or parallel trial; and (vi) peer review, original, full text studies. Exclusion criteria: (i) Soccer players with health problems (e.g., injuries, recent surgery) or another sports than soccer; (ii) Acute intervention or no HT-based programmes; (iii) Absence of control group; (iv) Non-controlled trial; (v) review, letter to editor, trial registration, proposal for protocols, editorial, book chapters and conference abstracts.
Information sources: Eletronic databases (PubMed, Web of Science, Scopus and Google scholar) were searched for relevant publications.

Main outcome(s): The maximal strength, power, sprint, change of direction and jump were chosen as the main outcome.

Additional outcome(s): None.

Quality assessment / Risk of bias analysis: The Tool for the assEssment of Study qualiTy and reporting in EXercise (TESTEX) scale was used to assess the risk of bias and methodological quality of the included studies (SMART et al. 2015). The TESTEX scale consists of 15-point and 12 items designed for assessing study quality (5points) and for reporting (10-points).

Strategy of data synthesis: Pre-training and post-training means and standard deviations (SD) were used to calculate effect sizes (ES) for dependente varibles. The ES values are presented with $95 \%$ confidence intervals (Cl). Calculated effect size (ES) were interpreted using the following scale: $\mathbf{0 . 0}-0.2$, trivial; $>0.2-0.6$, small, >0.6-1.2, moderate; >1.2-2.0, large; $>2.0-4.0$, very large; $>4.0$, extremely large (Hopkins, 2002). Heterogeneity was assessed using the $I^{2}$ statistic, with values of $75 \%$ interpreted as low, moderate and high heterogeneity, respectively (Higgins et al., 2003; Higgins, 2008). All analyses were carried out using the RevMan (Review Manager Version 5.4). Statistical significance was set at $p \leq 0.05$.

Subgroup analysis: None.

Sensitivity analysis: None.

Language: English.

Country(ies) involved: Brazil; and EUA.

Keywords: Football; sport science; sport performance.

Contributions of each author:

Author 1 - Jonatas Ferreira da Silva Santos - Lead the project, run the data search, 
performed the methodological assessment, conducting data extraction, analyse and interpreted the data, wrote and revised the original manuscript.

Email: jonatas_contato@hotmail.com

Author 2 - Moisés Luiz Martins conducting data extraction and wrote the manuscript.

Email: mlmprevi@gmail.com

Author 3 - Fabiano Trigueiro Amorim interpreted the data, wrote and revised the original manuscript.

Email: amorim@unm.edu

Author 4 - Marco Fabrício Dias Peixoto interpreted the data, wrote and revised the original manuscript.

Email: marcofabridp@gmail.com

Author 5 - Fernando Gripp - Run the data search, performed the methodological assessment, conducting data extraction, analyse and interpreted the data, wrote and revised the original manuscript.

Email: fernando.gripp@ufvjm.edu.br 